\title{
Ideal Magnetohydrodynamic Simulations of Low Beta Compact Toroid Injection into a Hot Strongly Magnetized Plasma
}

\author{
Wei Liu ${ }^{1}$, Scott C. Hsu ${ }^{2}$, Hui Li $^{1}$ \\ 1 Theoretical Division, Los Alamos National Laboratory, Los Alamos, NM, USA, \\ 87545 \\ ${ }^{2}$ Physics Division, Los Alamos National Laboratory, Los Alamos, NM, USA, 87545 \\ E-mail: wliu@lanl.gov
}

\begin{abstract}
We present results from three-dimensional ideal magnetohydrodynamic simulations of low $\beta$ compact toroid (CT) injection into a hot strongly magnetized plasma, with the aim of providing insight into CT fueling of a tokamak with parameters relevant for ITER (International Thermonuclear Experimental Reactor). A regime is identified in terms of CT injection speed and CT-to-background magnetic field ratio that appears promising for precise core fueling. Shock-dominated regimes, which are probably unfavorable for tokamak fueling, are also identified. The CT penetration depth is proportional to the CT injection speed and density. The entire CT evolution can be divided into three stages: (1) initial penetration, (2) compression in the direction of propagation, and reconnection with the background magnetic field, and (3) coming to rest and spreading in the direction perpendicular to injection. Tilting of the CT is not observed due to the fast transit time of the $\mathrm{CT}$ across the background plasma.
\end{abstract}

PACS numbers: 25.60.Pj, 28.52.Cx, 52.30.Cv,52.55.Fa,52.65.Kj

Submitted to: Nuclear Fusion 


\section{Introduction}

It is important to deliver fuel into the core of a tokamak fusion plasma to maintain steady-state operation, achieve more efficient utilization of deuterium-tritium fuel, and optimize the energy confinement time [1]. Several fueling schemes have been proposed, such as edge gas puffing, pellet injection [2], and compact toroid (CT) injection [3]. Among them, CT fueling is considered to be the most promising method for core fueling because the injection speed via this method is far higher than those of the other methods. Although extensive worldwide efforts have been devoted to study CT fueling theoretically [1, 3, 4], numerically [5, 6, 7, 8], and experimentally $[9,10,11,12,13,14,15,16,17,18,19,20,21,22,23,24,25,126$, 27, the dynamics of core CT fueling of large devices like ITER (International Thermonuclear Experimental Reactor) [28] is not well understood. CT injection has the potential to deposit fuel in a controlled manner at any point in the machine, from the edge to the core. Tangential (toroidal) injection can impart momentum for improving plasma $\beta$ and stability [29]. In a burning plasma device with only radio-frequency (rf) for auxiliary current drive, a CT

injection system may be the only internal profile control tool for optimizing bootstrap current and maintaining optimized fusion burn conditions. CT fueling also provides a good chance to study core transport in present machines, helium ash removal, and Edge Localized Mode (ELM) 30] control.

In this work, we employ a simple idealized model of a low $\beta$ CT propagating into a uniform slab plasma with a uniform magnetic field perpendicular to the CT injection direction, mimicking CT fueling into a tokamak with infinite aspect ratio. This model helps us identify different regimes of operation in terms of CT injection speed, density, and magnetic field strength, as well as understand the essential physics occurring during CT injection. More realistic scenarios, including the use of realistic tokamak profiles and geometry in the background plasma, as well as high $\beta$ CT's and dense plasma jets, are planned for follow-on research. Compared to past work on CT injection simulations, we have investigated new regimes especially in terms of higher injection velocity and a more ITER-relevant ratio (at least for low $\beta$ CT's such as spheromaks) of CT-tobackground magnetic field $(\sim 0.1)$. Simulations with higher injection velocity were made possible by the shock-handling capability of our three-dimensional (3D) ideal magnetohydrodynamic (MHD) code [31]. The lower CT-to-background magnetic field ratios, compared to past work, was enabled by the higher resolution of our code which allowed the boundary layer between the CT and background plasma to be properly resolved.

The paper is organized as follows. In Sec. 2, we describe the the problem setup including initialization of the CT and background slab plasma and the numerical model. We present the simulation results in Sec. 3, and our conclusions and implications for future CT fueling experiments are given in Sec. 4. 


\section{Problem setup and numerical model}

A low $\beta$ CT with spherical radius $r_{b}=1$, centered initially at $x_{b}=0, y_{b}=0$ and $z_{b}=z_{b, 0}=-12$, is injected along the $z$ axis into a lower density background plasma with injection velocity $v_{\text {inj }}$ (see Figure 1). The basic model assumptions and numerical treatments are briefly summarized here; they are essentially the same as those in Li et al. 32] where more details are given. This code uses high-order Godunovtype finite-volume numerical methods. These methods conservatively update the zoneaveraged fluid and magnetic field quantities based on estimated advective fluxes of mass, momentum, energy, and magnetic field at the zone interface [32]. The divergence-free condition of the magnetic field is ensured by a constrained transport (CT) scheme [33. All simulations were performed on the parallel Linux clusters at Los Alamos National Laboratory. The nonlinear system of time-dependent ideal MHD equations in 3D Cartesian coordinates $(x, y, z)$ is given here:

$$
\begin{array}{ll}
\frac{\partial \rho}{\partial t}+\nabla \cdot(\rho \vec{v}) & =0 \\
\frac{\partial(\rho \vec{v})}{\partial t}+\nabla \cdot\left(\rho \vec{v} \vec{v}+\left(p+\frac{B^{2}}{2}\right) \mathbf{I}-\vec{B} \vec{B}\right) & =0 \\
\frac{\partial E}{\partial t}+\nabla \cdot\left[\left(E+p+\frac{B^{2}}{2}\right) \vec{v}-\vec{B}(\vec{v} \cdot \vec{B})\right] & =0 \\
\frac{\partial \vec{B}}{\partial t}-\nabla \times(\vec{v} \times \vec{B}) & =0
\end{array}
$$

in which $\rho, p, \vec{v}, \vec{B}$ and $\mathrm{E}$ are the density, (gas) pressure, flow velocity, magnetic field, and total energy, respectively. $I$ is the unit diagonal tensor. The total energy is $E=p /(\gamma-1)+\rho v^{2} / 2+B^{2} / 2$, where $\gamma$ is the ratio of the specific heats. Note that a factor of $\sqrt{4 \pi}$ has been absorbed into the scaling for both the magnetic field $\vec{B}$ and current density $\vec{j}$. It should be noted that the details of effects such as reconnection and heat evolution could not be addressed accurately due to the ideal MHD model and the use of a simplified energy equation.

It is well established empirically in coaxial gun spheromak experiments that, under proper conditions, a spheromak "magnetic bubble" (a low $\beta \mathrm{CT}$ ) will be formed by the gun discharge [34. In our simulations, we do not model the CT formation process and instead start with a pre-formed CT moving toward the background slab plasma at speed $v_{\text {inj. }}$. The stationary background plasma is composed of a slab plasma confined by a uniform background magnetic field $B_{p}$ in the $x$ direction. For simplicity, we assume the background plasma has uniform initial number density $\rho_{p}=0.1$ and uniform initial temperature $T_{p}=0.1$.

In the simulations reported here, the CT structure is similar to the one given in Liu et al. [35]. The density profile of the CT plasma with radius $r_{b}=1$ is given by

$$
\rho_{b} \propto r_{c}^{2} \exp \left[-r_{c}^{2}-\left(z_{c}-z_{b}\right)^{2}\right]
$$

up to a normalization coefficient and a uniform temperature $T_{b}$, where $r_{c}=\sqrt{x^{2}+y^{2}}$ 
and $z_{c}=z$ (see Figure 1). The density profile used here has its peak shifted from the center of the CT, approximating a spheromak.

The CT magnetic field is determined by three key quantities: the length scale of the bubble magnetic field $r_{B}=2$, the amount of poloidal flux $\Psi_{p}$, and the index $\alpha$, which is the ratio of the CT toroidal to poloidal magnetic fields. For simplicity, the CT magnetic field $\vec{B}_{b}$ is also assumed to be axisymmetric. The poloidal flux function $\Psi_{p}$ is specified as

$$
\Psi_{p} \propto r_{c}^{2} \exp \left[-r_{c}^{2}-\left(z_{c}-z_{b}\right)^{2}\right] .
$$

The poloidal fields, up to a normalization coefficient, are

$$
B_{b, r_{c}}=-\frac{1}{r_{c}} \frac{\partial \Psi_{p}}{\partial z_{c}}, \quad B_{b, z_{c}}=\frac{1}{r_{c}} \frac{\partial \Psi_{p}}{\partial r_{c}},
$$

while the toroidal magnetic field is

$$
B_{b, \varphi_{c}}=\frac{\alpha \Psi_{p}}{r_{c}}=\alpha r_{c} \exp \left[-r_{c}^{2}-\left(z_{c}-z_{b}\right)^{2}\right] .
$$

The azimuthal component of the CT Lorentz force is zero, but the total azimuthal Lorentz force due to the combined fields and currents of the CT may be non-zero.

The CT also has uniform injection velocity $v_{\text {inj }}$ and uniform rotation angular speed $\omega$. In this paper the ratio of the CT's toroidal to poloidal magnetic fields $\alpha$, the rotation speed of the CT $\omega$, and the specific heat $\gamma$ are taken to be $\sqrt{10}, 0$ and $5 / 3$, respectively. Physical quantities are normalized by the characteristic system length scale $R_{0}=10 \mathrm{~cm}$, density $\rho_{0}=7.77 \times 10^{-9} \mathrm{~g} / \mathrm{cm}^{-3}$ (corresponding to plasma number density, which is the sum of electron and ion number density, of $18.6 \times 10^{14} \mathrm{~cm}^{-3}$ ), and velocity $V_{0}=1.7 \times 10^{8} \mathrm{~cm} \mathrm{~s}^{-1}$. Other quantities are normalized as: time $t=1$ gives $R_{0} / V_{0}=5.9 \times 10^{-8} \mathrm{~s}$, magnetic field $B=1$ gives $\left(4 \pi \rho_{0} V_{0}^{2}\right)^{1 / 2}=5.3 \times 10^{4} \mathrm{G}$, and energy $E=1$ gives $\rho_{0} V_{0}^{2} R_{0}^{3}=2.24 \times 10^{11}$ ergs.

The boundary conditions are all perfectly conducting in the $y$ and $z$ directions except at the port where the $\mathrm{CT}$ is injected, while in the $x$ direction outflow boundary conditions are employed in order to mimic the toroidal geometry of a tokamak. Since the toroidal dimension is much larger than the poloidal dimension in a real tokamak and we focus on the early stage of the CT evolution before one toroidal propagation time of the Alfvén wave induced by the CT propagation, the out-flowing boundary condition is more appropriate than the periodic boundary condition used in Suzuki et al. [7]. In order to minimize the influence of the entrance port, the port will be switched on when the top of the CT reaches the bottom boundary at $t=2 / v_{\text {inj }}$ and it will be switched off at $t=5 / v_{\text {inj }}$ after the CT has fully entered the computation domain $\left(t \sim 4 / v_{\text {inj }}\right)$. Suzuki et al. have pointed out that the boundary condition in the background magnetic field $(x)$ direction is important, i.e., magnetic reconnection has more influence on CT deceleration with perfectly conducting boundary conditions than with stress-free boundary conditions such as outflow and periodic boundary conditions [7]. The total computational domain is $|x| \leq 9,|y| \leq 9$, and $|z| \leq 9$, corresponding to a $(180 \mathrm{~cm})^{3}$ box in actual length units (assuming the physical dimension of the 
injected CT radius $r_{b}=10 \mathrm{~cm}$ ). The numerical resolution used here is $400 \times 400 \times 800$, where the grid points are assigned uniformly in the $x, y$, and $z$ directions. A cell $\delta x$ $(=\delta y=2 \delta z=0.045)$ corresponds to $0.45 \mathrm{~cm}$. Since the plasma skin depth and ion gyroradius based on the parameters of ITER are no more than $\delta x$, the simulations based on an MHD model are appropriate.

\section{Results}

In this section we present ideal MHD simulation results on the injection of a low $\beta$ CT into a hot strongly magnetized plasma. We organize our results into three topics: (1) parameter regimes, in terms of $v_{\text {inj }}$ and ratio of CT-to-background magnetic field, of CT evolution including the identification of a promising regime for ITER-relevant precise core fueling, (2) detailed description of the CT evolution for the ITER-relevant regime, and (3) dependence of the $\mathrm{CT}$ penetration depth on $v_{\text {inj }}$ and the initial $\mathrm{CT}$ density.

\subsection{Parameter regimes of $C T$ injection}

Based on our simulation results, we find that the evolution of the injected CT depends predominantly on the initial injection speed $v_{\text {inj }}$ and the initial ratio of CT-tobackground magnetic field. As shown in Figure 2, there are several qualitatively distinct regimes of CT injection in terms of the above two parameters.

First, below a threshold injection speed $V_{\mathrm{L}}$, the $\mathrm{CT}$ is unable to penetrate the background plasma at all (see left panel of Figure 3). The conducting sphere (CS) model [1] requires that the initial CT kinematic energy exceeds the background magnetic field energy excluded by the CT volume,

$$
\frac{1}{2} \rho_{b} v_{\mathrm{inj}}^{2}>\frac{1}{2} B_{p}^{2} \rightarrow v_{\mathrm{inj}}>V_{\mathrm{AC}}=\frac{B_{p}}{\sqrt{\rho_{b}}} .
$$

Our results show that $V_{\mathrm{L}}$, which is inferred $(\sim 0.5)$ from extrapolation of the data shown in Fig. 9(a), is less than $V_{\mathrm{AC}}$. This is because of the compression of the CT during the penetration, which is ignored by the CS model (see discussion of \$3.2), i.e., the CT actually excludes less volume than its initial volume, therefore leading to a smaller initial injection speed threshold. And since this compression is related to the background plasma magnetic field (see discussion in $\$ 3.2$ ), this injection speed threshold is thus dependent on the background plasma magnetic field. However, $V_{\mathrm{AC}}$ derived from the CS model still gives a rough estimate of the lower limit of the injection speed needed for penetration.

Second, above a different threshold injection speed $V_{\mathrm{AP}}$, a strong shock and wavefront are observed to develop ahead of the CT, dominating the system evolution (see middle panel of Figure 3). This threshold is determined by the condition when $v_{\text {inj }}$ exceeds the Alfvén speed of the background plasma, i.e., $v_{\text {inj }}>V_{\mathrm{AP}}=B_{p} / \sqrt{\rho_{p}}$. This shock/wavefront-dominated regime is probably not favorable for CT fueling because it 
may strongly perturb the tokamak equilibrium. Also, in this case the CT deposition is highly nonlocal (middle panel of Figure 3). For comparison, the result $\left(v_{\text {inj }}=1.1\right)$ without shock/wavefront development is also presented (right panel of Figure 3) $\left(V_{\mathrm{AP}}=3.16\right.$ in this case).

Third, in the regime $V_{\mathrm{L}}<v_{\text {inj }} \lesssim V_{\mathrm{AP}}$, CT evolution is further determined by the ratio $S_{r}=B_{b} / B_{p}$ of the CT field $B_{b}$ to the background plasma magnetic field $B_{p}$. If $S_{r} \gg 10$, a large non-zero initial Lorentz force results in strong CT expansion that also leads to the development of a shock and a wavefront that dominate the system evolution [35]. As stated above, this does not favor controlled plasma fueling. In the regime $1<S_{r} \lesssim 10$, which is the regime Suzuki et al. have discussed extensively [5, 6, 7], the $\mathrm{CT}$ is decelerated by both the magnetic pressure and magnetic tension forces. In this regime, the $\mathrm{CT}$ tilts while reconnection occurs between the $\mathrm{CT}$ and background plasma magnetic fields. Suzuki et al. proposed the Non-slipping Conducting Sphere (NS) model [5, 6, 7], which matches their simulation results of vertical injection without a magnetic field gradient pretty well [7]. Our simulation results in this regime verify their conclusions. Because it is difficult for the CT field $B_{b}$ generated by a co-axial gun to be larger than the tokamak field $B_{p}$ of several Tesla or more, it is important to explore the regime $S_{r}<1$, which is the primary focus of the remainder of this paper. We have identified this regime (right panel of Figure 31) as a promising one for CT fueling of ITER-relevant plasmas due to the precise spatial deposition of the CT and the deep penetration that can be achieved for core fueling applications. In this paper, we have focused on background plasmas with $\beta_{p}=0.02$ like in ITER, while simulation results with $\beta_{p}=0.2$ like in the National Spherical Torus Experiment (NSTX) will be studied in follow-on work.

\section{2. $C T$ Evolution}

In this sub-section, we describe in detail the CT evolution for the ITER-relevant regime of $V_{\mathrm{L}}<v_{\text {inj }} \lesssim V_{\mathrm{AP}}$ and $S_{r}<1$. In this regime, the $\mathrm{CT}$ evolution can be divided into three stages: (1) initial CT penetration, (2) CT compression in the propagation direction $(z)$ and reconnection, and (3) coming to rest and spreading in the $x$ (toroidal) direction. Magnetic reconnection, starting late in the second stage in our simulations, arises due to numerical diffusion and leads to mixing between the CT and background plasmas.

In order to mimic CT injection with ITER-relevance, we adopt physical quantities as given in Table1 1 and choose the injection speed $v_{\text {inj }}$ such that $V_{\mathrm{AC}}=1.0<v_{\text {inj }}=1.1<$ $V_{\mathrm{AP}}=3.16$ (this falls into the "ITER-relevant" case of Figure 22). Figure 4 displays the time evolution of the plasma density (color contours in common logarithmic scale) in the $x-z$ plane with $y=0$. The white solid contour lines indicate the magnetic pressure $p_{B}=B^{2} / 2$. Fig. 5 displays the axial profile of $x$ and $y$ integrated density $\int_{x} \int_{y} \rho d x d y$ at different times corresponding to Fig. 4 . The entire evolution can be described by the three stages mentioned above. 
Figure 6 displays the magnetic field $B_{x z}$, (arrows) and current density $j_{y}$ (color contours) in the $x-z$ plane at $y=0$. During the first stage (initial penetration), the CT experiences a very strong magnetic obstacle, and therefore the CT plasma is highly compressed and the plasma density increases at the interface between the CT and background plasmas. A large plasma current also appears at the interface due to the compression of the background magnetic field seen in Figure 6(a). This current sheet is bent and broken into two parts (Figure 6(b)) due to the magnetic field configuration of the CT field. Some reconnection takes place at the left part of the current sheet as shown in Figure 7. The CT is successively decelerated by the magnetic tension force of the background magnetic field.

After the CT has fully entered the background plasma region (after $t \sim 4$ ), the background field lines, some of which have reconnected with the CT magnetic field at the CT's leading edge, are reconnected again with the CT magnetic field at the CT's trailing edge, as seen in Figure 6(c). As pointed out by Suzuki et al. [5], via this process the CT is separated from the background fields lines, through which the magnetic tension force decelerating the $\mathrm{CT}$ is relaxed. At $t=4.375$, a magnetic configuration schematically shown in Figure 7 is formed. This interpretation is supported by Figure 8 , which displays the axial distributions of density and $B_{x}$. From Figure 8 , we can see that $B_{x}$ changes sign from positive to negative and then to positive again. The transition from positive to negative happens at larger $z$ on the left hand side while at smaller $z$ on the right hand side, which is the case in Figure 7 (right) (see two dashed lines in Figure 7 (right)). From Figure 7, primary reconnection sites are at the upper left and lower right sections of the CT. The reconnection process allows the high-density CT plasma to escape from the CT and eventually flow outward along the background magnetic field horizontally (Figure 7). The CT plasma starts to contract in $z$ and expand in $x$. The reconnection is asymmetric about the CT axis, and this asymmetry results in CT plasma outflow in a direction that is not completely in the $x$ direction, but rather obliquely (panel (d) of Figure 6). Eventually it will become more parallel to $x$ (right panel of Figure 3 ). Magnetic flux is being destroyed as well. During this process the MHD wave drag from Alfvén waves induced by horizontal plasma outflow [1, 4] might further slow down the CT plasma. Note that Suzuki et al. [7] showed that the CT penetration depth, based on a model with magnetic tension force as the main deceleration mechanism, matches simulation results very well, implying that MHD wave drag forces may not be important in CT deceleration. The initially injected magnetic and perpendicular kinematic energies are converted into parallel kinematic energy. Contrary to [1] and [5, 6, 7], CT tilting, the time scale of which is proportional to $\sqrt{B_{b} B_{p} / \rho_{b}}$, is not observed in our case due to the fast injection and short $\mathrm{CT}$ transit time as required for the ITER-relevant regime (Table 1).

After the high-density CT plasma has been depleted during the compression stage (after $t \sim 9$ ), the CT field diminishes and only the slightly perturbed background field survives, almost orienting to the direction of the initial background magnetic field (see Figure 4 and Figure 6(d)). Almost all of the initially injected magnetic and kinematic 
energy now survive in the form of parallel kinematic energy. The CT comes to rest in the injection direction $(z)$. A narrow elongated structure along $x$ results, as seen in the lower middle and lower right panels of Figure 4. This line-shaped structure with a spread of only $\Delta z \sim 0.2$ (much smaller than the size of the background plasma, see also Fig. (5) implies a very precise fuel deposition capability.

\subsection{CT penetration depth}

In this sub-section, we establish the dependence of $\mathrm{CT}$ penetration depth, an important parameter for CT fueling of tokamaks, on the experimentally controllable parameters of CT injection speed $v_{\text {inj }}$ and initial density $\rho_{b}$, which collectively determine the initial CT energy. The penetration depth $S$ is defined as the axial $(z)$ distance between the final mean position of the injected $\mathrm{CT}$ and the injection location on the boundary. We find that $S$ is highly dependent on the initial injection speed $v_{\text {inj }}$ and CT density $\rho_{b}$. Figure 9(a) displays the relationship between the $S$ and $v_{\text {inj }}$, showing that $S$ is proportional to $v_{\text {inj }}$ if $V_{\mathrm{L}}<v_{\text {inj }}<V_{\mathrm{AP}}$. Figure 9(b) displays the relationship between the CT density and penetration depth, which shows that the penetration depth $S$ increases with the CT density. The CT spread in the $z$ direction is around $\sim 0.2$ in all cases (see also Fig. (5), which is very small compared to the background plasma size $\sim 20$. These two empirical relationships are important in the sense that they provide clues for how to choose the injection speed and CT density to get precise fuel deposition, therefore controlling the core plasma profile in a large tokamak such as ITER. It is very hard to manage that by other methods such as pellet injection [26].

For the case shown in Figure 9(b), the parameters given in Table 1 are adopted $\left(v_{\text {inj }}=2.0\right)$ except CT density $\rho_{b}$ and CT field $B_{b}$. We choose them such that the plasma parameter $\beta=0.2$ is kept constant for all the simulation results shown in Figure 9(b). We keep $\beta$ constant since experimentally this is more reasonable than changing CT density or CT field strength independently. Thus, increasing CT density means increasing both the initial kinematic energy $E_{k}=\int 1 / 2 \rho_{b} v_{\mathrm{inj}}^{2} d V$, where $d V$ is the infinitesimal CT volume, and initial magnetic energy $E_{m}=\int 1 / 2 B_{b}^{2} d V$. However, because of the limiting criterion given in Eq. 8, the initial CT kinematic energy $E_{k}$ should be much larger than the initial CT magnetic energy $E_{m}$ since the background field is much larger than the CT field in all simulation results presented in Figure 9(b) $\left(B_{b} / B_{p}=0.1\right)$. Also the residual initial total force $\mathbf{j} \times \mathbf{B}-\nabla p$ is proportional to the CT density $\sim O\left(\rho_{b}\right)$. Therefore, increasing the CT density $\rho_{b}$ would elevate the initial residual force, which would result in shock/wavefronts. Thus, with too large a density ratio $\left(\rho_{b} / \rho_{p} \gtrsim 15\right.$, which is inferred from simulation results), the fueling is not as localized as the case with smaller CT density, while generating similar phenomena as the shock/wavefront dominated cases.

Results of Figure 9 remind us of the importance of initially injected energy upon the penetration depth. As discussed in $\$ 3.2$, how long the compression stage lasts is determined by the sum of the initially injected kinematic and magnetic energy. The 
larger the sum is, the longer it will take for the reconnection to dissipate/convert the energy. If we assume that the deceleration mechanism of the CT is mainly due to the magnetic tension force, which is independent of the initially injected energy [7], then the larger initially injected energy would lead to deeper fueling given the same injection speed $v_{\text {inj }}$ (since plasma parameter $\beta \ll 1$, the internal energy of the CT plasma can be ignored). Certainly the larger injection speed would lead to deeper penetration.

Figure 10 shows the time evolution of the net toroidal magnetic flux $\psi_{t}=\int B_{y} d S$ (only positive $B_{y}$ is selected) with $v_{\text {inj }}=1.1$ and $\rho_{b} / \rho_{p}=10$. Before $t=6.25$, the net CT toroidal magnetic flux increases due to the initial penetration and compression of the magnetic field lines at the interface of the CT and background plasma. After $t=11.25$, the line structure reaches the horizontal boundaries and some toroidal magnetic flux flows out. Between $t=7.5$ and $t=11.25$, the only source of the destruction of the net toroidal magnetic flux is magnetic reconnection due to the numerical diffusion. If we fit the decay as $\psi_{t}(t) / \psi_{t}(t=0) \equiv \exp \left(-t / \tau_{\text {res }}\right)$, the "resistive" dissipation time due to numerical diffusion is $\tau_{\text {res }} \sim 10.9$. Therefore the global resistive decay due to numerical diffusion is not important on the time scales of CT transit time $(t \sim 5)$ given injection speed $v_{\text {inj }}=1.1$. In the simulation, mixing arises from numerical diffusion induced reconnection and occurs on the same time scale as the CT transit time. However, the mixing should be less of a factor in reality due to the much smaller diffusion in a high temperature tokamak.

\section{Conclusions \& discussion}

In this paper we presented nonlinear ideal MHD simulation results of a compact toroid injected into a hot strongly magnetized plasma. The simulations are intended to provide insights into CT fueling of an ITER-class tokamak. As a first step, we have investigated the problem of a high density low $\beta$ CT injected into a slab background plasma with uniform magnetic field and density. We intend to investigate the injection of high $\beta$ CT's as well as unmagnetized dense plasma jets, and also incorporate more realistic background profiles in follow-on work.

Our main findings are as follows. A regime is identified in terms of CT injection speed and CT-to-background magnetic field ratio that appears promising for precise core fueling. Shock-dominated regimes, which are probably unfavorable for tokamak fueling, are also identified. The CT penetration depth is proportional to the CT injection speed and density. For the regime identified as favorable for precise core fueling, the entire CT evolution can be divided into three stages: (1) initial penetration, (2) compression in the direction of propagation, and reconnection with the background plasma, and (3) coming

to rest and spreading in the direction perpendicular to injection. Tilting of the CT is not observed due to the fast transit time of the CT across the background plasma. Reconnection occurring at the upper left and lower right portions of the CT fragments the CT and leads to CT plasma outflow horizontally along the background magnetic field lines, forming a line-shaped structure almost parallel to the background magnetic 
field. We have also studied how the penetration depth is determined and have found an empirical relationship between the penetration depth versus the initial injection speed and CT plasma density. The penetration depth is proportional to the injection speed and CT density. When the CT comes to rest, it is highly compressed in the direction of injection, implying that very precise deposition is possible.

In a device with high bootstrap current fraction, optimized density and pressure profiles must be maintained. A core fueling system is all that a burning plasma may have to alter core plasma conditions and for burn control. Initial density peaking via core fueling provides more flexibility to reach ignition. If a CT injection system is within the ITER-relevant, no-shock/wavefront regime as required by $v_{\text {inj }} \ll V_{\text {AP }}, \rho_{b} / \rho_{p} \lesssim 15$ and $B_{b} / B_{p} \ll 1$, then precise core deposition with minimal background equilibrium perturbation may be possible.

Because $V_{\mathrm{AC}} \sim 1700 \mathrm{~km} \mathrm{~s}^{-1}$ for ITER (using parameters in Table 1), it will be a challenge for present CT injectors with $v_{\text {inj }} \sim 400 \mathrm{~km} \mathrm{~s}^{-1}$ to achieve deep penetration. A potential solution is to increase $\rho_{b} / \rho_{p}$ to at least 100 , which would reduce $V_{\mathrm{AC}}$ to a more reasonable value $\left(\sim 170 \mathrm{~km} \mathrm{~s}^{-1}\right)$. Due to computational limitations, a simulation of such a case (very dense CT) is beyond the scope of this study. However, to provide insight into the penetration characteristics of a very high density injected plasma, we report here some preliminary results on the injection of a dense $\left(\rho_{b} / \rho_{p}=538\right.$ or $\left.\rho_{b} \sim 10^{17} \mathrm{~cm}^{-3}\right)$, cool $\left(T_{b} / T_{p}=3.33 \times 10^{-4}\right.$ or $\left.T_{b}=2.5 \mathrm{ev}\right)$, and very weakly magnetized plasma [37], which would be an interesting alternative to CT injection. Figure 11 suggests that this type of injected plasma is capable of reaching the core of the tokamak, albeit with worse localization as discussed in Sec. 3.3. This will be reported on in more detail in follow-on work.

\section{Acknowledgments}

The authors thank Shengtai Li for extensive advice on the code. The authors also thank Roger Raman and Xianzhu Tang for very useful discussions and constructive comments. This work was funded by DOE contract no.DE-AC52-06NA25396 under the Los Alamos

Laboratory Directed Research and Development (LDRD) Program.

[1] P. B. Parks. Phys. Rev. Lett., 61:1364, 1988.

[2] S. L. Milora et al. Phys. Rev. Lett., 42, 1979.

[3] L. J. Perkins et al. Nucl. Fusion, 28:1365, 1988.

[4] W. A. Newcomb. Phys. Fluids B, 3:1818, 1991.

[5] Y. Suzuki et al. Physics of Plasmas, 7:5033, 2000.

[6] Y. Suzuki et al. Nuclear Fusion, 40:277, 2000.

[7] Y. Suzuki et al. Nuclear Fusion, 41:873, 2001.

[8] Y. Suzuki et al. Nuclear Fusion, 41:769, 2001.

[9] M. R. Brown and P. M. Bellan. Phys. Rev. Lett., 64:2144, 1990.

[10] M. R. Brown and P. M. Bellan. Nucl. Fusion, 32:1125, 1992.

[11] R. Raman et al. Phys. Rev. Lett., 73:3101, 1994.

[12] R. Raman et al. Nuclear Fusion, 37(7):967-72, 1997.

[13] R. Raman et al. In in Controlled Fusion and Plasma Physics Proceedings of 24th Europe Conference, Berchtesgaden, 1997, volume 21A, Part I. Europe Physical Society, Geneve, 1999. 
[14] M. J. Gouge et al. In Proceedings of 16th International Conference, Montreal, 1996, volume 3. International Atomic Energy Agency, Vienna, 1997.

[15] N. Fukumoto et al. In Bull. Amer. Phys. Soc., volume 42, page 1961, 1997.

[16] J. T. Slough and A. L. Hoffman. In Fusion Energy 1996 (Proc. 16th Int. Conf. Montreal, 1996), Vol. 2, p. 237 IAEA, Vienna (1997).

[17] R. Décoste et al. In Fusion Energy 1998 (Proc. 17th Int. Conf. Yokohama, 1998) (Vienna: IAEA) CD-ROM file and http://www.iaea.org/programmes/ripc/physics/start.htm.

[18] R. Raman and P. Gierszewski. Fusion Eng. Des., 39-40:977-85, 1998.

[19] D. Q. Hwang et al. Phys. Plasmas, 6:1515, 1999.

[20] T. Ogawa et al. Nuclear Fusion, 39(11Y):1911-1915, 1999.

[21] D. Q. Hwang et al. Nucl. Fusion, 40:897, 2000.

[22] J. T. Slough and A. L. Hoffman. Phys. Plasmas, 6:253, 1999.

[23] C. Xiao et al. Phys. Plasmas, 11:4041, 2004.

[24] M. Nagata et al. Nucl. Fusion, 45:1056, 2005.

[25] D. Liu et al. Nucl. Fusion, 46:104, 2006.

[26] G. Olynyk and J. Morelli. Nuclear Fusion, 48:095001, 2008.

[27] R. Raman. Fusion Engineering and Design, 83:1368-1374, 2008.

[28] International Atomic Energy Agency. ITER technical basis. Technical report, International Atomic Energy Agency, 2001. No 19 in the ITER EDA Documentation Series.

[29] R. Raman. Fusion Science and Technology, 50:84, 2006.

[30] F. Wagner et al. In Proceedings of the 13th Conference on Plasma Physics and Controlled Nuclear Fusion Research, volume 1. IAEA, Vienna, 1982.

[31] Shengtai Li et al. ApJS, 174:1-12, 2008.

[32] H. Li et al. Astrophys. J., 643:92-100, 2006.

[33] D. S. Balsara, and D. S. Spicer. J. Comput. Phys., 149:270, 1999.

[34] P. M. Bellan. Spheromaks. Imperial College Press, London, 2000.

[35] W. Liu et al. Phys. Plasmas, 15:072905, 2008.

[36] R. Raman et al. Fusion Technol., 24:239, 1993.

[37] F. D. Witherspoon et al. submitted to Rev. Sci. Instrum., 2009.

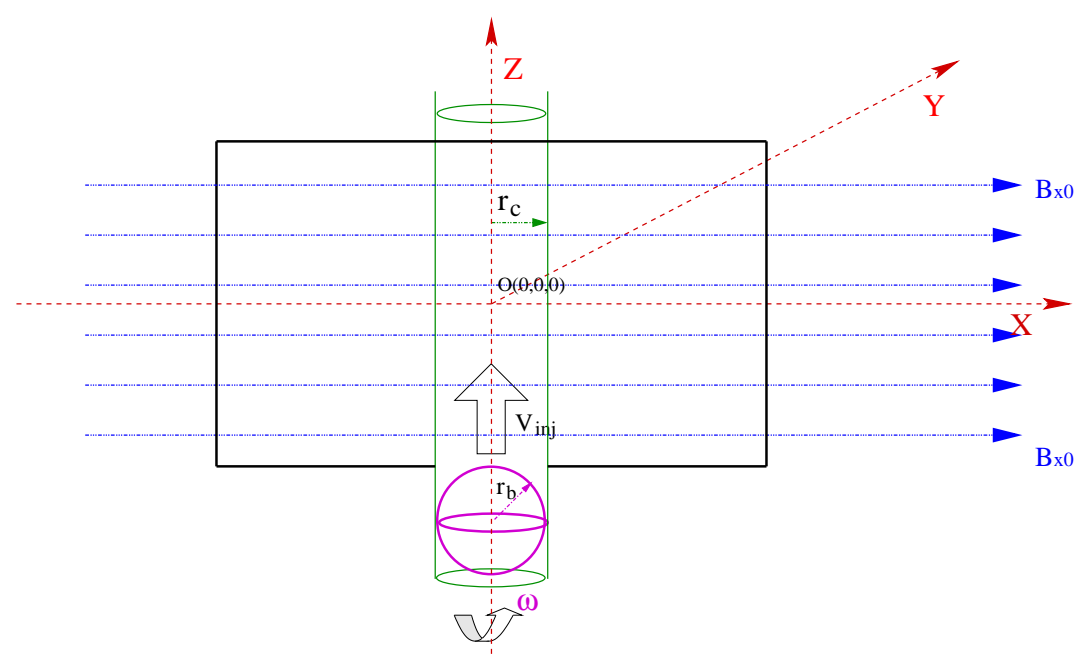

Figure 1. Schematic of the simulation geometry showing the coordinate system. In the text, the direction along the $z$-axis is defined as the axial direction. 


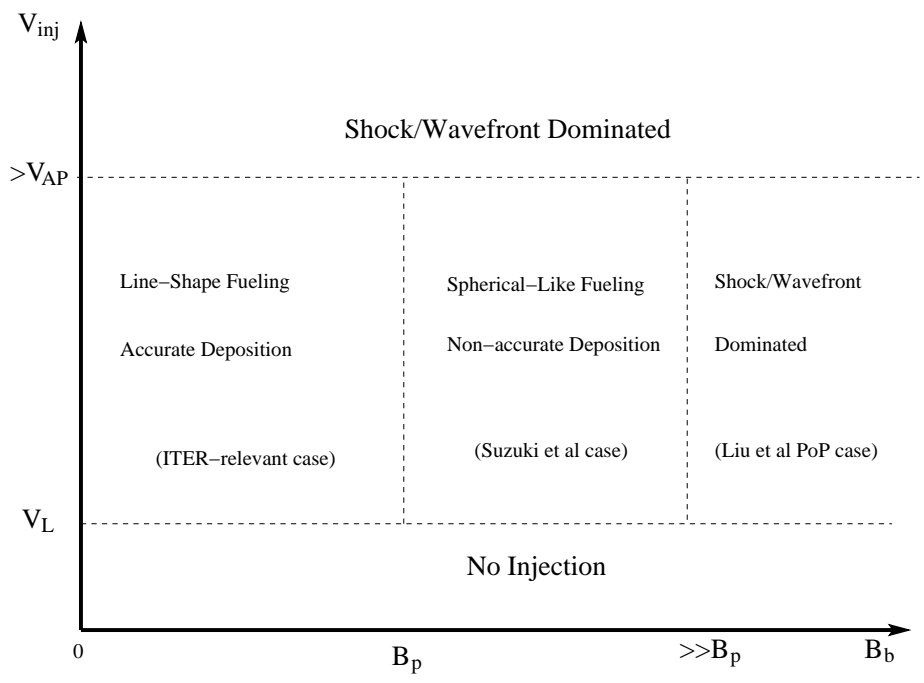

Figure 2. Qualitative behavior for CT injection in terms of CT injection speed and magnetic field strength.
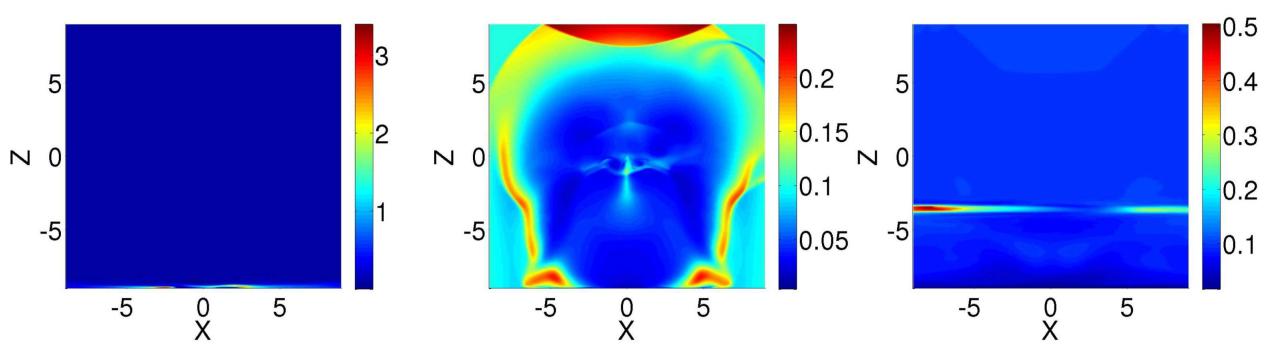

Figure 3. (color) Density in the $x-z$ plane at $y=0$. Left panel: at $t=20$ with injection speed $v_{\text {inj }}=0.3$, which shows no penetration. Middle panel: at $t=2.5$ with injection speed $v_{\text {inj }}=10.0$, which shows non-localized deposition. Right panel: at $t=20$ with injection speed $v_{\text {inj }}=1.1$, which shows highly localized deposition. $V_{\mathrm{AP}}=3.16$.

Table 1. Normalized physical quantities. Note that $V_{\mathrm{AC}}=B_{p} / \sqrt{\rho_{b}}=1.0$. Injection speed $v_{\text {inj }}$ is between $V_{\mathrm{L}}$ and $V_{\mathrm{AP}}$. Plasma is assumed to be composed of half deuterium and half tritium. The plasma density is the sum of electron and ion densities.

\begin{tabular}{ccccc}
\hline & \multicolumn{2}{c}{ CT } & \multicolumn{2}{c}{ Background } \\
\hline Physical Quantities & numerical & physical & numerical & physical \\
\hline Magnetic Field & $B_{b}=0.1$ & $0.53 \mathrm{~T}$ & $B_{p}=1.0$ & $5.3 \mathrm{~T}$ \\
Density & $\rho_{b}=1.0$ & $1.86 \times 10^{15} \mathrm{~cm}^{-3}$ & $\rho_{p}=0.1$ & $1.86 \times 10^{14} \mathrm{~cm}^{-3}$ \\
Temperature & $T_{b}=0.001$ & $75 \mathrm{ev}$ & $T_{p}=0.1$ & $7.5 \mathrm{kev}$ \\
plasma $\beta=2 \rho T /<B^{2}>$ & \multicolumn{2}{c}{$\beta_{b}=0.2$} & \multicolumn{2}{c}{$\beta_{p}=0.02$} \\
Alfvén Speed $V_{\mathrm{A}}=B / \sqrt{\rho}$ & $V_{\mathrm{AB}}=0.1$ & $170 \mathrm{~km} \mathrm{~s}^{-1}$ & $V_{\mathrm{AP}}=3.16$ & $5.4 \times 10^{3} \mathrm{~km} \mathrm{~s}^{-1}$ \\
Sound Speed $V_{c}=\sqrt{\gamma T}$ & $V_{\mathrm{cB}}=1.7 \times 10^{-3}$ & $2.89 \mathrm{~km} \mathrm{~s}^{-1}$ & $V_{\mathrm{cP}}=0.41$ & $7.0 \times 10^{2} \mathrm{~km} \mathrm{~s}^{-1}$ \\
\hline
\end{tabular}




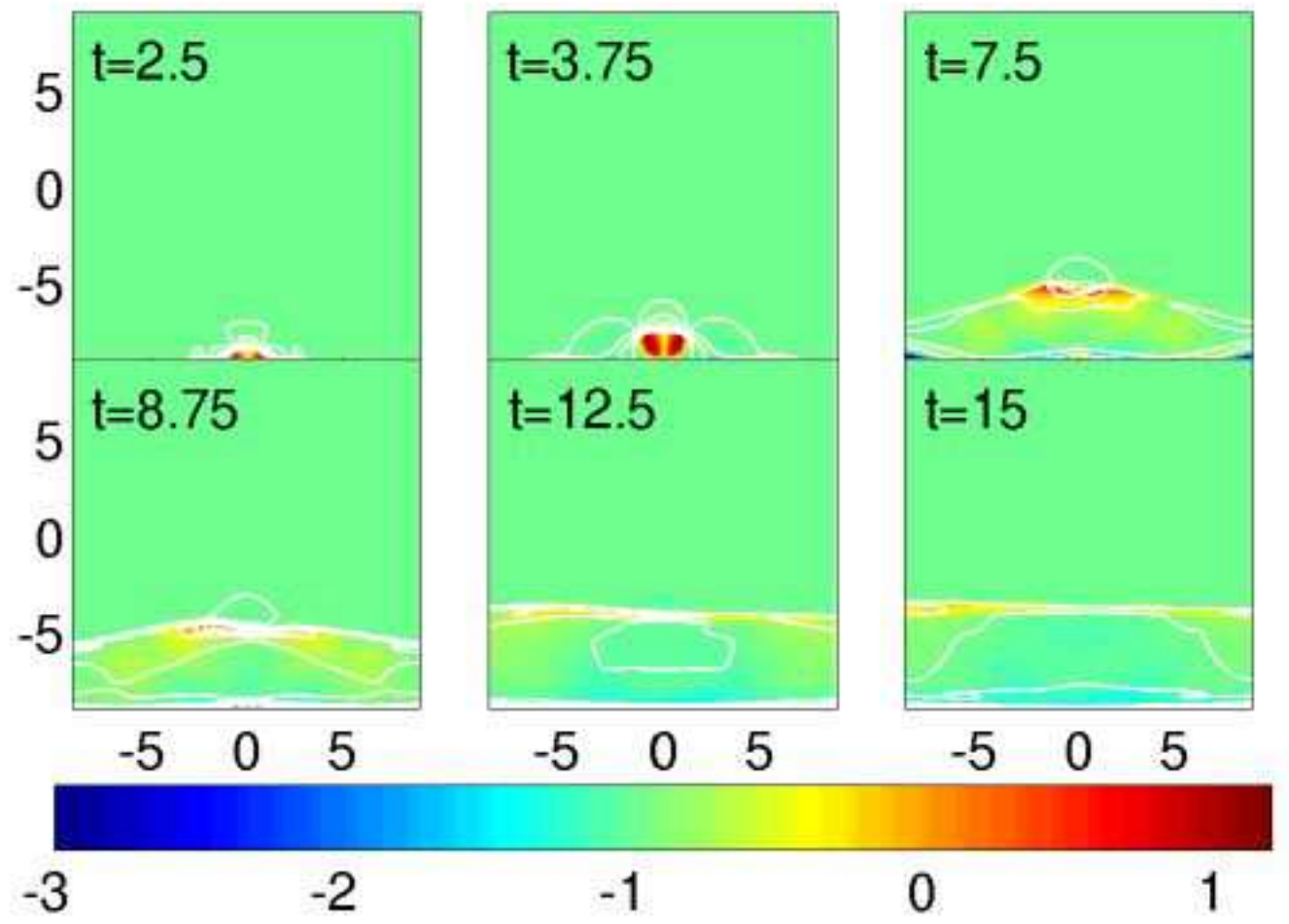

Figure 4. (color) Density (logarithmic scale) in the $x-z$ plane at $y=0$ as a function of time. The white lines are contours of magnetic pressure $p_{B}=B^{2} / 2$. For $t=2.5$, $p_{B} \in[0.0,1.6843]$; for $t=3.75, p_{B} \in[0.0,1.7612]$; for $t=7.5, p_{B} \in[0.0,1.4405]$; for $t=8.75, p_{B} \in[0.0,1.1872]$; for $t=12.5, p_{B} \in[0.0,1.0219]$; for $t=15, p_{B} \in[0.0,1.008]$. 


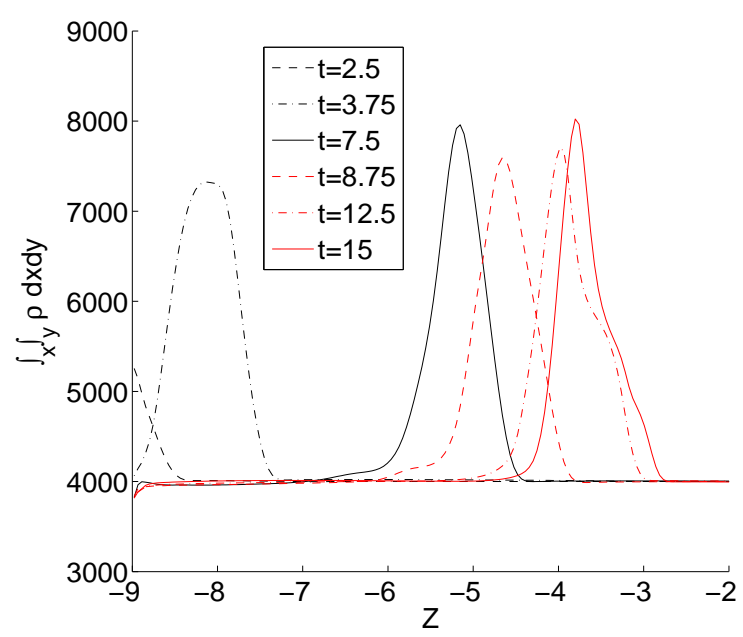

Figure 5. (color) $x$ and $y$ integrated density $\int_{x} \int_{y} \rho d x d y$ versus $z$ at different times corresponding to Fig. 4 Black dash: $t=2.5$, black dash dot: $t=3.75$; black solid: $t=7.5$; red dash: $t=8.75$; red dash dot: $t=12.5$, red solid: $t=15$.

(a)

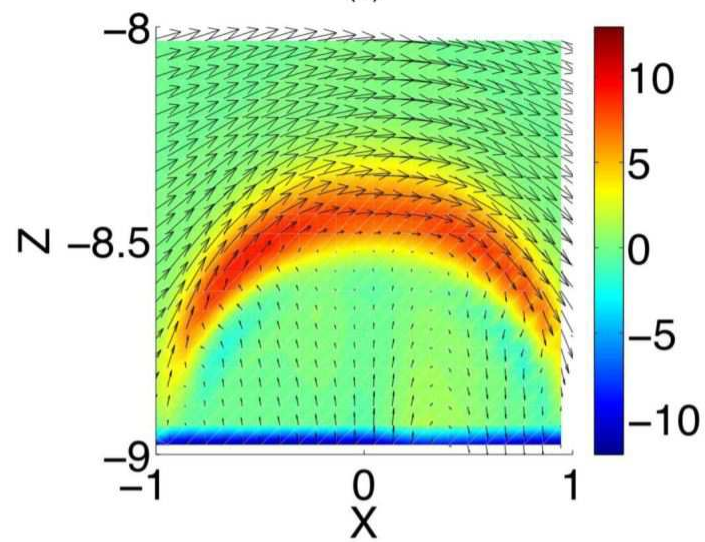

(c) (b)

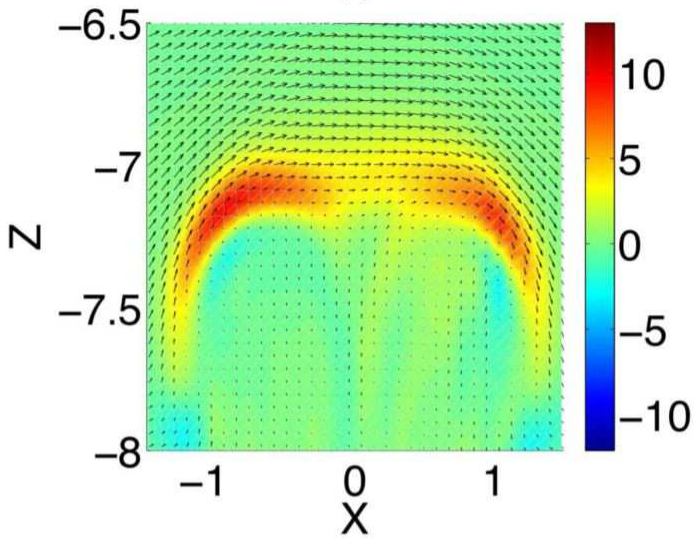

(d)

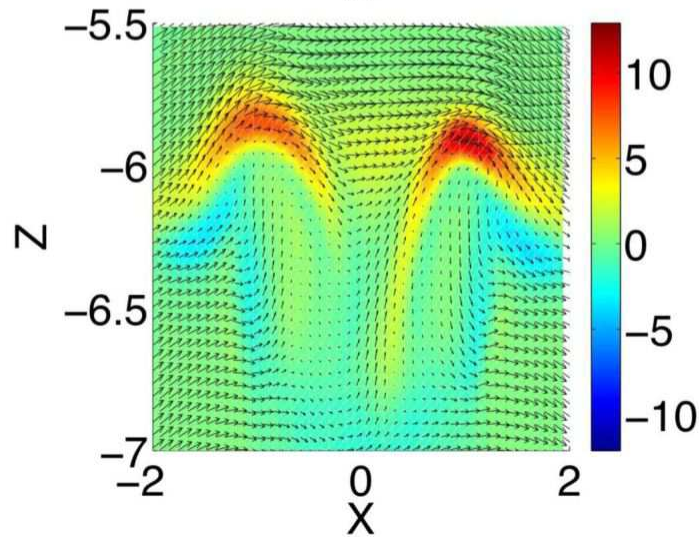

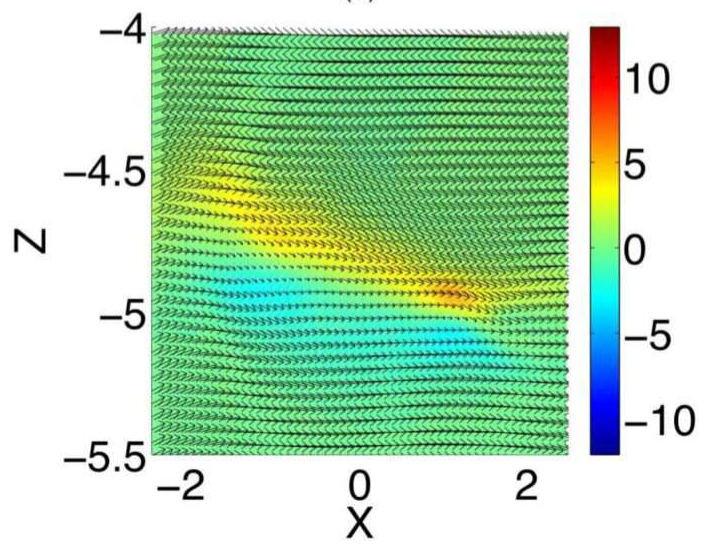

Figure 6. (color) Magnetic field and current density $j_{y}$ in the $x$ - $z$ plane as a function of time at $y=0$. The color contours show $j_{y}$ while arrows show $B_{x}$ and $B_{z}$, which are normalized to their maximum values. (a) $t=2.5$; (b) $t=4.375$; (c) $t=6.25$; (d) $t=8.75$. Please note that the scales for the abscissa and ordinate are not identical. 

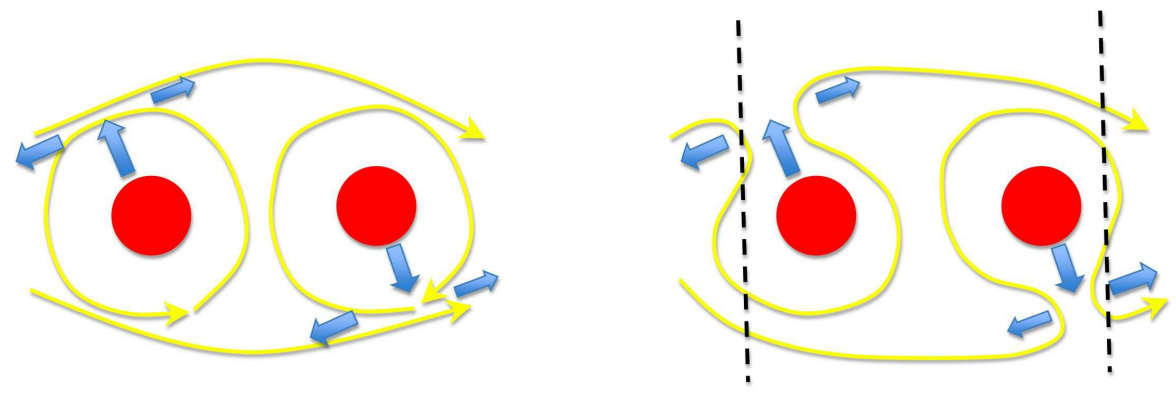

Figure 7. (color) Diagram of the magnetic configuration in the contraction stage. Red color indicates the high-density CT plasma. Yellow lines indicate magnetic field lines, while blue arrows indicate CT plasma flow patterns. See Figure 8 for dotted black lines.
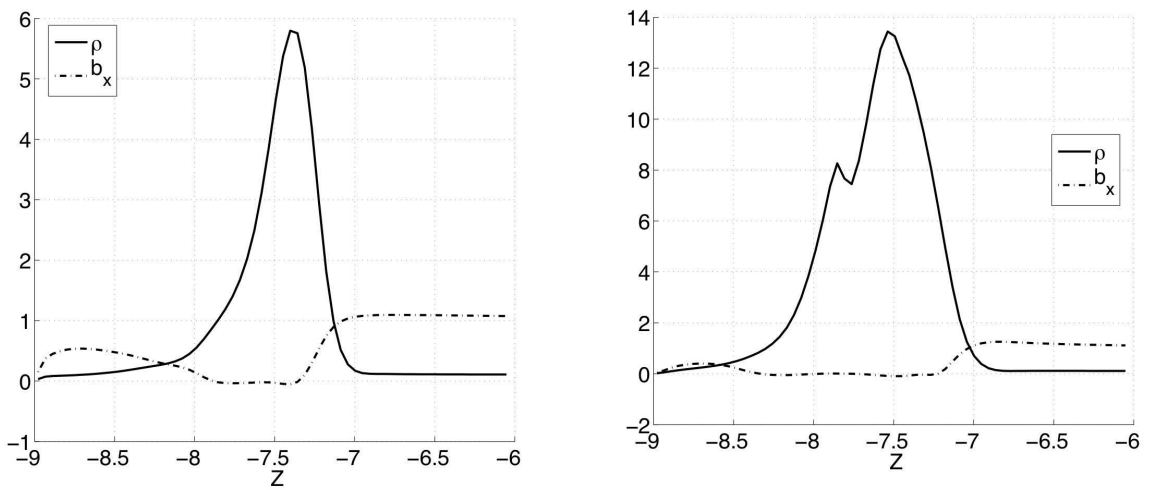

Figure 8. Axial profiles of several quantities at $t=4.375$ with $(x, y)=(-1.125,0)$ (left) and $(x, y)=(0.855,0)$ (right) . The density $\rho$ and magnetic field strength in the $x$-direction $B_{x}$ are shown for evaluating the magnetic configuration shown in Figure 7 (right). These two axial profiles correspond to the two dash lines in Figure 7 (right). 
(a)

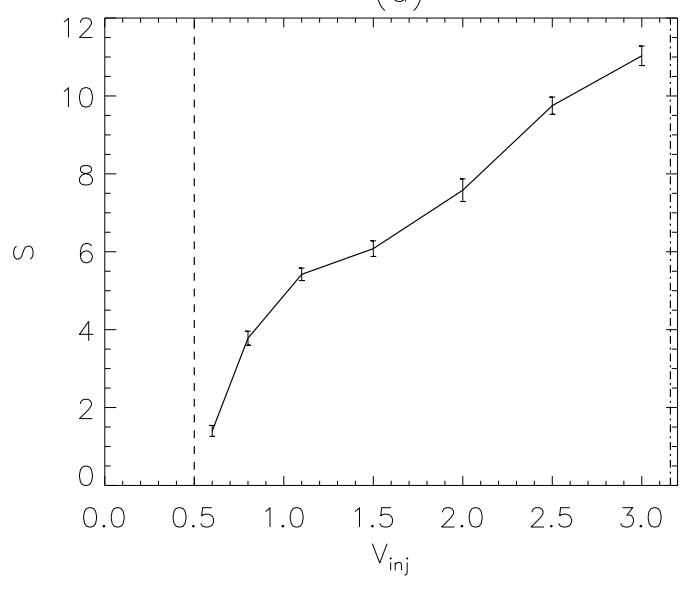

(b)

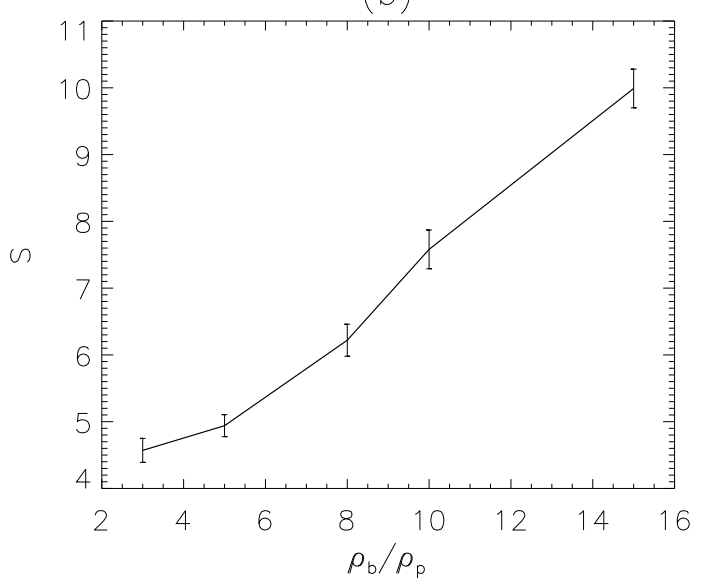

Figure 9. (a) Penetration depth $S$ versus injection speed $v_{\text {inj }}$ with $\rho_{b} / \rho_{p}=10$ and (b) Penetration depth $S$ versus CT density $\rho_{b}$ with $v_{\text {inj }}=2.0$. Both panels assume $B_{b} / B_{p}=0.1$. The penetration depth is defined as the distance between the final mean position of the injected plasma and the bottom boundary. The error bar indicates the final size of the injected $\mathrm{CT}$ in the $z$-direction. In panel (a), the dash line indicates $V_{\mathrm{L}} \sim 0.5$ and the dash dot line indicates $V_{\mathrm{AP}}=3.16$.

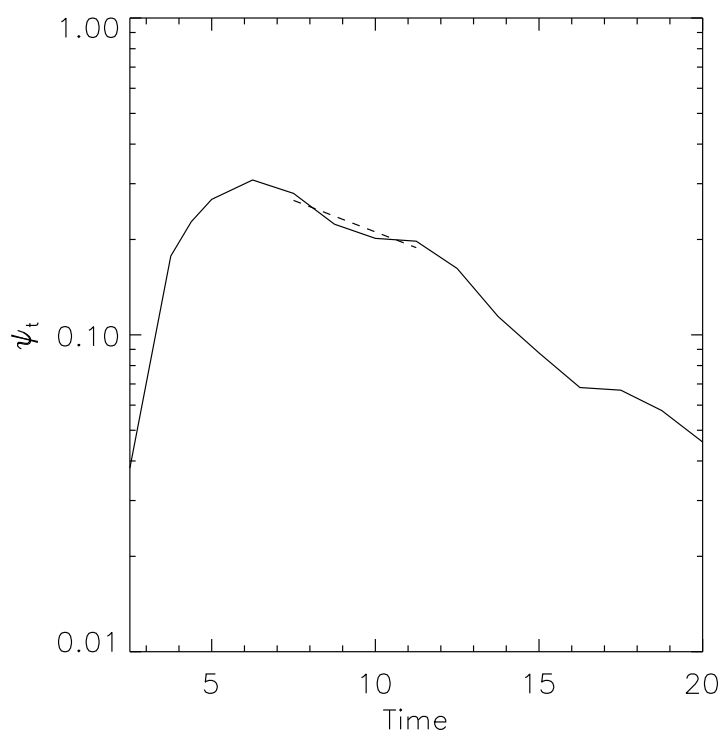

Figure 10. Decay of the net toroidal magnetic flux $\psi_{t}=\int B_{y} d S$, where only positive $B_{y}$ is selected with $v_{\mathrm{inj}}=1.1$ and $\rho_{b} / \rho_{p}=10$. The dashed line fits the data between $t=7.5$ and $t=11.25$ with the formula $\psi_{t}(t) / \psi_{t}(t=0) \equiv \exp \left(-t / \tau_{\text {res }}\right)$, where $\tau_{\text {res }}=10.9$ is the resistive dissipation time due to numerical diffusion, and it is much larger than the CT transit time $\sim 5$ given injection speed $v_{\text {inj }}=1.1$. 


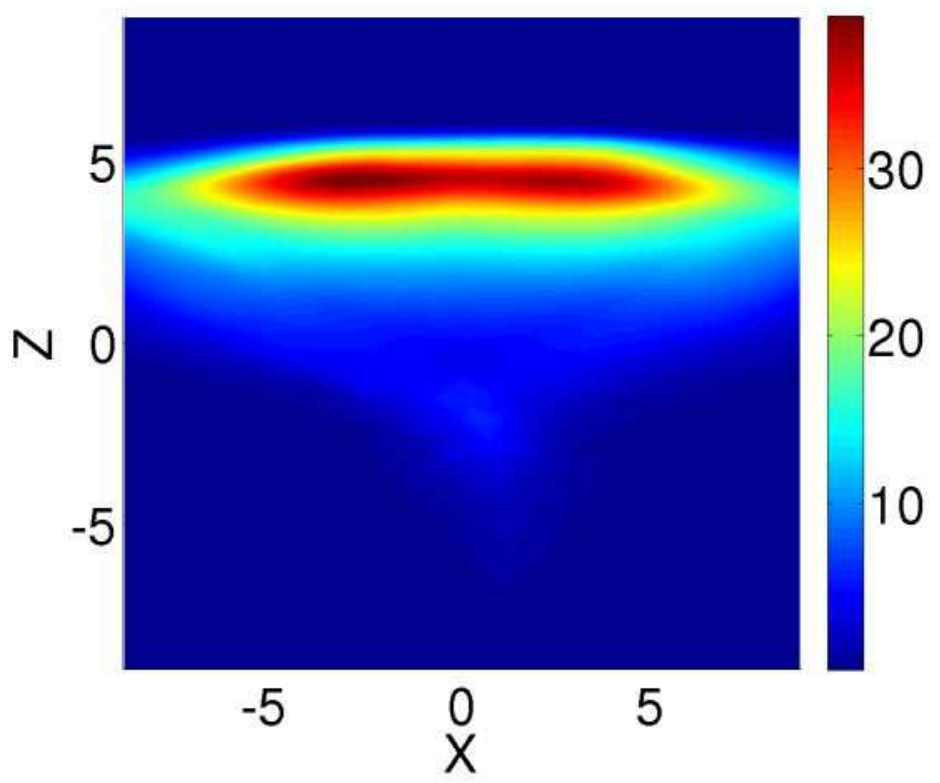

Figure 11. (color) Density in the $x-z$ plane of $y=0$ at $t=150$ with injection speed $v_{\text {inj }}=0.12, \rho_{b} / \rho_{p}=538$, and $B_{b} / B_{p}=0.001$, which shows worse localized deposition. Please note that the CT is still moving with almost the initial injection speed. 